\title{
Search for second overtone mode Cepheids in Magellanic Clouds
}

\section{Characteristics of second overtone mode pulsations ${ }^{\star}$}

\author{
E. Antonello, D. Fugazza, and L. Mantegazza \\ Osservatorio Astronomico di Brera, via E. Bianchi 46, 23807 Merate, Italy \\ Received 23 August 2001 / Accepted 17 January 2002

\begin{abstract}
The analysis of CCD observations of ten new suspected second overtone mode Cepheids in the SMC does not confirm the nature of such stars; most of them appear to be first overtone mode Cepheids. A careful analysis of the OGLE best second overtone mode candidates and of the second overtone component of double mode Cepheids in SMC and LMC has been performed in order to clarify the possible effects of a resonance between pulsation modes. There are some indications that such a resonance is indeed operating in these pulsators. A brief discussion of the effects of blending on the light curve shape is also reported, and the usefulness of the Fourier parameters as mode discriminators is discussed.
\end{abstract}

Key words. stars: oscillations - stars: variables: Cepheids - galaxies: Magellanic Clouds

\section{Introduction}

The importance of second overtone $(2 O)$ mode Cepheids is due to their being, among Cepheids, another possible benchmark for the stellar interior and evolution theory other than fundamental and first overtone mode Cepheids. The existence of $2 O$ pulsations in these stars was predicted by Stobie (1969) and its first indication was the discovery of the double-mode Cepheid CO Aur (Mantegazza 1983) which pulsates simultaneously in the first and second overtone modes. One of the many by-products of the MACHO, EROS and OGLE projects was the discovery of many double-mode Cepheids (DMCs) pulsating both in the fundamental- and first-overtone mode $(F / 1 O)$, and the first- and second-overtone $(1 O / 2 O)$ modes in the LMC and SMC (e.g. Alcock et al. 1995; Beaulieu et al. 1995; Beaulieu et al. 1997; Udalski et al. 1999a; Soszynski et al. 2000). The MACHO team also searched for pure $2 O$ Cepheids and found one potential candidate in the LMC (Alcock et al. 1999), while the OGLE team found a sample of thirteen pure $2 O$ candidates in the SMC (Udalski et al. 1999b). In the meanwhile, Antonello \& Kanbur (1997) studied the $2 O$ pulsation characteristics produced by nonlinear models; more recently Bono et al. (2001) considered the stability and nonlinear characteristics, and the possibility of using $2 O$ Cepheids as distance indicators.

Send offprint requests to: E. Antonello, e-mail: elio@merate.mi.astro.it

* Based in part on observations collected at ESO-La Silla.
Antonello \& Kanbur (1997) noted in particular the possible effects of the resonance $P_{2} / P_{6}=2$ at $P_{2} \lesssim 1 \mathrm{~d}$ ( $P$ is the period) between the second and sixth overtone mode. Resonances represent a powerful comparison tool between observations and theoretical model predictions, because they affect the shape of the curves of stars in a specific period range, for example $P_{0} / P_{2}=2$ at $P_{0} \sim$ $10 \mathrm{~d}$ in fundamental mode Cepheids (Simon \& Lee 1981) and $P_{1} / P_{4}=2$ at $P_{1} \sim 3 \mathrm{~d}$ in first overtone Cepheids (Antonello et al. 1990). The close comparison allows us to probe the stellar interior and to put constraints on the stellar physical parameters (see for example Feuchtinger et al. 2000).

In a previous paper (Mantegazza \& Antonello 1998) we reported about the search for $2 O$ candidates in the SMC. In the present paper we report the observational results for another ten candidates which were suggested by Beaulieu (1997); moreover we discuss the observed properties of the pure $2 O$ candidates and $2 O$ components of DMCs, with the purpose of finding further evidence of the expected resonance phenomena.

\section{Observations}

The observations were performed with the direct CCD camera at the Dutch $0.91 \mathrm{~m}$ telescope of La Silla (ESO) during three runs in November 1997 (6 nights), July 1998 (12 nights) and October 1998 (15 nights). The CCD detector was the ESO chip No. 33, which is a TEK CCD with $512 \times 512$ pixels, pixel size of $27 \mu \mathrm{m}$ and spatial resolution of 0 '. 44 , providing a small field of view of $3 ! 77 \times 3 ! 77$. 
Table 1. Number of frames.

\begin{tabular}{llll}
\hline Field & $V$-frames & $I$-frames & Number of Stars \\
\hline 0 & 46 & 17 & 3169 \\
5 & 44 & 16 & 2657 \\
9 & 51 & 16 & 3268 \\
$11 \mathrm{a}$ & 48 & 15 & 3131 \\
$11 \mathrm{~b}$ & 43 & 15 & 2788 \\
13 & 41 & 16 & 2834 \\
$15 \mathrm{a}$ & 45 & 17 & 2783 \\
$15 \mathrm{~b}$ & 44 & 17 & 2624 \\
\hline
\end{tabular}

Table 2. External error estimate.

\begin{tabular}{llll}
\hline$V$ & $\sigma$ & $V$ & $\sigma$ \\
\hline 16.0 & 0.017 & 20.0 & 0.092 \\
17.0 & 0.020 & 20.5 & 0.135 \\
18.0 & 0.023 & 21.0 & 0.193 \\
18.5 & 0.028 & 21.5 & 0.268 \\
19.0 & 0.040 & 22.0 & 0.362 \\
19.5 & 0.061 & & \\
\hline
\end{tabular}

8 fields were selected in order to observe the ten second overtone candidates; the selection was optimized in order to include as much as possible other Cepheids detected by the EROS project, so that an homogeneous comparison between the light curves of different types of Cepheids was possible. In this way other 46 known $F$ and $1 O$ Cepheids were observed. In the first two runs, because these observations were performed in the context of other projects, only one or two $V$ images per night were obtained, while in the third run, which was fully dedicated to this project, up to three $V$ images per night were obtained, along with an $I$ image. The exposure times were $600 \mathrm{~s}$ for both filters. For each field, we got from 41 to $51 \mathrm{~V}$ images and from 15 to $17 I$ images. The detailed number of observations are reported in Table 1 . Bias and twilight flat field frames were obtained in each useful night. The identification number of each field corresponds to the one adopted by the EROS project for the respective enclosing field.

\section{Data reduction and analysis}

Bias subtraction and flat field corrections were performed using the IRAF package (Tody 1993). It was necessary to correct the images for the slightly different pointing of the telescope and orientation of the CCD camera in the different runs. The stellar photometry was performed by means of the IRAF/DAOPHOT package (Stetson 1987; Davis 1994). First, the $V$ images were measured. A list of reliable stars were prepared by selecting the common objects in the best four images of each field. The number of objects measured in each field is reported in the 4th column of Table 1. The brightness of these stars was then measured in all the frames with the technique of "fixed position photometry" (Kaluzny et al. 1998). The same procedure was applied to $I$ frames. The transformations from instrumental to standard $V$ and $I$ magnitudes were performed by means of the observations of two Landolt fields (1992). We then computed for each field the standard

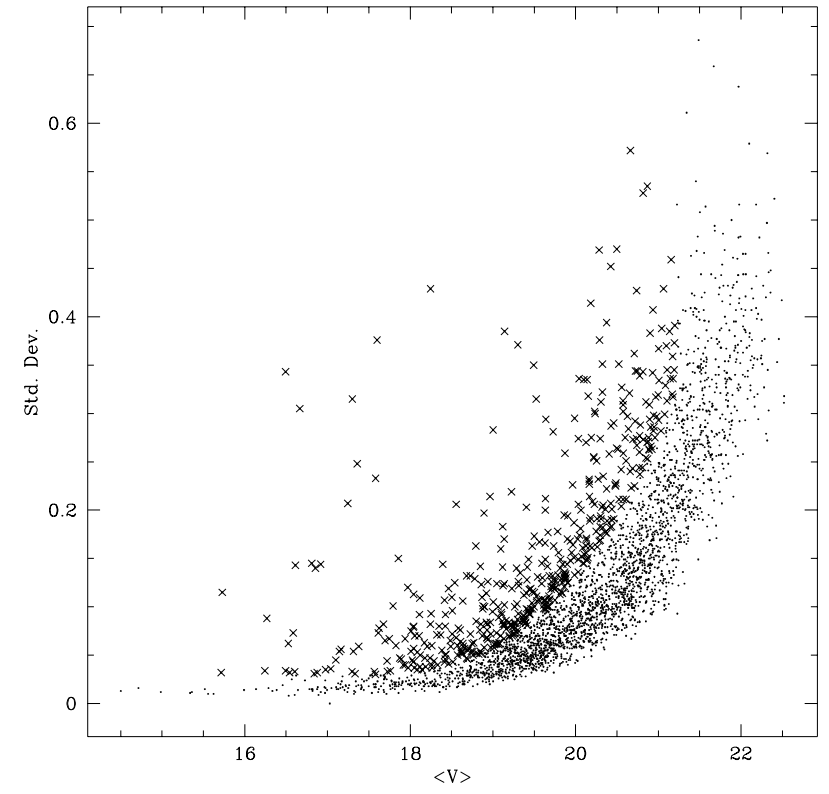

Fig. 1. Standard deviations of the $V$ measurements of all the observed stars in Field 0 (dots) vs. their average values. The brightest stars have measurements with accuracies of few hundredths of a magnitude. The crosses indicate the stars investigated for variability.

deviations of the star brightness about the mean value. As an example, in Fig. 1 we report the standard deviations ( $V$ data) for field 0 . From these diagrams we can estimate the external errors of our measurements, which are summarized in Table 2, and furthermore we can see that the limiting $V$ magnitude is about 22.0; analogous figures were obtained for the $I$ frames.

An independent search for variable stars in these fields was performed using $V$ data. We selected as candidate variable stars in each field all the objects with a standard deviation larger than $3 \sigma$ times the median standard deviation of the objects with the same mean magnitude. In these way a number of objects between 250 and 550 were selected for each field. Their light curves were then inspected in detail by computing the leastsquares power spectra and phasing the data according to the most likely periods; we estimate that the lowest detectable amplitude is of about $0.15 \mathrm{mag}$ for $V \sim 19$. As a result, about 400 variable stars were detected; they include Cepheids, RR Lyr stars, W Vir, Eclipsing Binaries, Long Period Variables and a few suspected high amplitude $\delta$ Scuti stars. In the present paper we will discuss the properties of Cepheids.

\section{4. $F, 1 O$ and DM Cepheids}

A detailed list of the 69 Cepheids detected is reported in Tables 3 and 4, which include the following relevant characteristics: equatorial coordinates, mean $V$ and $I$ magnitudes, $P$, pulsation mode and the corresponding EROS and OGLE identification number if the star was already 
Table 3. F, 1O, and DM Cepheids.

\begin{tabular}{|c|c|c|c|c|c|c|c|c|}
\hline Name & RA (2000) & Dec (2000) & $V$ & $I$ & $P[\mathrm{~d}]$ & mode & EROS & OGLE \\
\hline $11 b-2383$ & $0: 50: 56.32$ & $-73: 12: 21.0$ & 18.43 & 18.01 & 0.493 & $?$ & & \\
\hline 11b-2118 & 0:50:15.20 & $-73: 12: 45.0$ & 17.31 & 16.99 & 0.496 & $?$ & & \\
\hline $11 b-2740$ & 0:50:15.11 & $-73: 11: 18.9$ & 18.18 & 17.53 & 0.501 & $?$ & & \\
\hline 05-0076 & 0:53:08.89 & $-73: 20: 48.9$ & 18.05 & 17.46 & 0.614 & $1 O(\mathrm{DMC})$ & 05-06579 & 06-180332 \\
\hline $11 \mathrm{a}-2816$ & 0:49:31.29 & $-73: 09: 56.2$ & 17.24 & 17.04 & 0.748 & $?$ & & \\
\hline 11b-2003 & 0:50:30.39 & $-73: 09: 24.0$ & 18.03 & 17.38 & 0.756 & DMC & $11-03611$ & \\
\hline 00-0240 & $0: 43: 34.68$ & $-73: 08: 20.3$ & 18.04 & 17.38 & 0.787 & $1 O$ & 00-09087 & 03-088752 \\
\hline $13-1414$ & 0:53:19.91 & $-73: 06: 44.0$ & 18.58 & 17.84 & 0.898 & $?$ & & \\
\hline $11 a-0765$ & 0:50:02.38 & $-73: 08: 53.0$ & 17.95 & 17.34 & 0.910 & $1 O$ & $11-03635$ & 05-190752 \\
\hline 15b-0466 & $0: 57: 34.03$ & $-73: 17: 06.4$ & 17.45 & 16.95 & 1.019 & $1 O$ & $15-02299$ & 07-214519 \\
\hline $11 a-0164$ & 0:49:37.66 & $-73: 10: 34.2$ & 18.94 & 18.11 & 1.064 & $?$ & & \\
\hline $13-0578$ & 0:53:39.69 & $-73: 07: 38.0$ & 17.23 & 16.75 & 1.101 & $1 O$ & $13-08012$ & 06-199740 \\
\hline 05-1176 & $0: 53: 22.47$ & $-73: 19: 58.9$ & 17.32 & 16.71 & 1.117 & $1 O$ & 05-07604 & 06-180172 \\
\hline 11b-2129 & 0:50:50.82 & $-73: 12: 25.6$ & 18.11 & 17.06 & 1.134 & - & $11-00916$ & 05-266152 \\
\hline 15b-0121 & 0:58:15.79 & $-73: 18: 30.1$ & 17.96 & 17.42 & 1.238 & $F$ & $15-01538$ & \\
\hline 00-1809 & 0:44:03.88 & $-73: 06: 12.6$ & 16.92 & 16.38 & 1.295 & $1 O$ & & 03-148834 \\
\hline $15 a-1147$ & $0: 57: 38.60$ & $-73: 13: 18.0$ & 17.59 & 17.01 & 1.297 & $F$ & $15-05985$ & \\
\hline 09-0538 & 0:46:44.94 & $-72: 57: 35.6$ & 17.57 & 16.99 & 1.310 & $F$ & 09-10857 & $04-082233$ \\
\hline $11 \mathrm{a}-2864$ & 0:49:29.65 & $-73: 09: 05.1$ & 17.77 & 16.87 & 1.311 & $1 O$ & & 05-111714 \\
\hline 11b-1003 & 0:50:39.66 & $-73: 09: 39.7$ & 16.97 & 16.39 & 1.394 & $1 O$ & $11-03506$ & 05-190562 \\
\hline $15 a-0348$ & $0: 57: 35.03$ & $-73: 13: 03.8$ & 17.81 & 17.16 & 1.426 & $F$ & $15-06177$ & 07-217946 \\
\hline 11b-1137 & 0:50:45.31 & $-73: 12: 23.1$ & 17.04 & 16.30 & 1.440 & $1 O$ & $11-00859$ & 05-185163 \\
\hline $15 a-1156$ & $0: 57: 40.25$ & $-73: 13: 16.9$ & 17.02 & 16.34 & 1.465 & $F$ & $15-06041$ & \\
\hline 11b-1136 & 0:51:03.24 & $-73: 12: 38.7$ & 17.63 & 16.84 & 1.580 & $F$ & $11-01007$ & 05-266150 \\
\hline $00-1846$ & 0:44:13.12 & $-73: 05: 55.0$ & 16.82 & 16.26 & 1.593 & $1 O$ & & 03-148839 \\
\hline 00-0949 & 0:44:02.02 & $-73: 08: 24.7$ & 17.61 & 16.87 & 1.672 & $F$ & $00-09485$ & 03-148864 \\
\hline $13-1372$ & $0: 53: 57.82$ & $-73: 07: 02.0$ & 17.46 & 16.88 & 1.735 & $F$ & $13-08888$ & 06-277037 \\
\hline 09-0173 & $0: 47: 18.19$ & $-72: 59: 43.0$ & 17.09 & 16.38 & 1.761 & $1 O$ & 09-09408 & 04-131058 \\
\hline $11 \mathrm{a}-2408$ & 0:49:57.09 & $-73: 09: 50.2$ & 17.85 & 17.02 & 1.777 & $F$ & & \\
\hline 11a-2912 & $0: 49: 40.20$ & $-73: 11: 52.7$ & 17.53 & 16.92 & 1.783 & $F$ & $11-00261$ & 05-111570 \\
\hline 00-0228 & $0: 43: 55.70$ & $-73: 08: 23.1$ & 17.29 & 16.67 & 1.788 & $F$ & 00-09400 & \\
\hline 05-0094 & 0:53:08.89 & $-73: 20: 48.9$ & 17.75 & 16.94 & 1.800 & $F$ & $05-06783$ & 06-180338 \\
\hline 15b-1385 & 0:57:41.69 & $-73: 16: 01.8$ & 17.13 & 16.55 & 1.810 & $F$ & $15-03416$ & \\
\hline $00-2770$ & $0: 44: 14.48$ & $-73: 07: 32.4$ & 17.58 & 16.88 & 1.811 & $F$ & 00-10492 & 03-148895 \\
\hline 00-2639 & 0:44:01.48 & $-73: 05: 42.4$ & 16.86 & 16.19 & 1.833 & $1 O$ & & 03-148843 \\
\hline 00-0189 & $0: 43: 28.47$ & $-73: 08: 37.3$ & 17.36 & 16.66 & 1.854 & $F$ & $00-08706$ & 03-088671 \\
\hline 05-2302 & 0:53:18.90 & $-73: 17: 48.4$ & 17.36 & 16.68 & 1.873 & $F$ & 05-09664 & 06-185340 \\
\hline $11 \mathrm{a}-1087$ & 0:49:29.85 & $-73: 09: 17.6$ & 17.54 & 16.74 & 1.887 & $F$ & $11-02696$ & 05-111708 \\
\hline $15 b-2414$ & $0: 57: 49.15$ & $-73: 18: 24.0$ & 17.52 & 16.85 & 1.902 & $F$ & $15-01284$ & \\
\hline 11b-0043 & 0:51:03.01 & $-73: 12: 31.5$ & 18.03 & 17.06 & 1.913 & $F$ & & 05-266145 \\
\hline $11 \mathrm{a}-2411$ & 0:49:57.11 & $-73: 09: 40.5$ & 17.17 & 16.49 & 1.974 & $F$ & $11-02769$ & 05-111536 \\
\hline 05-1706 & 0:53:30.21 & $-73: 20: 33.2$ & 16.76 & 16.20 & 2.003 & $F$ & $05-07152$ & 06-180343 \\
\hline 05-1050 & $0: 53: 12.73$ & $-73: 20: 40.8$ & 16.84 & 16.14 & 2.020 & $1 O$ & $05-06778$ & 06-180157 \\
\hline 11a-1402 & 0:49:35.05 & $-73: 10: 52.8$ & 16.77 & 16.09 & 2.062 & $1 O$ & $11-01166$ & 05-111505 \\
\hline $15 a-0299$ & 0:58:03.16 & $-73: 13: 10.7$ & 16.22 & 15.71 & 2.107 & $1 O$ & $15-06474$ & \\
\hline $15 b-1857$ & $0: 57: 42.97$ & $-73: 16: 00.0$ & 16.92 & 16.25 & 2.136 & $F$ & $15-03457$ & \\
\hline 15b-1741 & 0:58:06.57 & $-73: 17: 05.2$ & 16.12 & 15.59 & 2.198 & $1 O$ & $15-02765$ & \\
\hline 11a-1922 & 0:50:00.12 & $-73: 09: 52.2$ & 16.88 & 16.17 & 2.351 & $F$ & $11-02625$ & 05-111529 \\
\hline 11a-0396 & $0: 49: 52.85$ & $-73: 09: 08.7$ & 17.52 & 16.72 & 2.492 & $F$ & 11-03212 & 05-111547 \\
\hline 13-0690 & 0:53:30.67 & $-73: 07: 01.9$ & 16.83 & 16.20 & 2.496 & $F$ & $13-08467$ & \\
\hline $15 a-1885$ & $0: 57: 48.14$ & $-73: 12: 12.9$ & 16.17 & 15.62 & 2.553 & $1 O$ & $15-07195$ & \\
\hline 11a-0262 & 0:49:36.79 & $-73: 10: 01.0$ & 17.17 & 16.85 & 2.568 & - & $11-02070$ & 05-111664 \\
\hline 11b-0307 & 0:51:00.75 & $-73: 10: 28.4$ & 16.35 & 15.65 & 2.569 & $1 O$ & $11-03048$ & 05-271149 \\
\hline $15 a-1309$ & $0: 58: 12.37$ & $-73: 12: 33.7$ & 16.36 & 15.82 & 2.818 & $F$ & $15-07204$ & \\
\hline 09-0436 & $0: 47: 21.87$ & $-72: 58: 13.1$ & 16.63 & 15.93 & 3.048 & $F$ & 09-10885 & 04-131025 \\
\hline 00-1105 & $0: 43: 37.34$ & $-73: 07: 43.1$ & 16.50 & 15.79 & 3.263 & $F$ & 00-09691 & 03-088650 \\
\hline $15 a-1797$ & $0: 57: 31.31$ & $-73: 13: 05.1$ & 16.21 & 15.51 & 3.372 & $F$ & $15-06092$ & 07-217862 \\
\hline $11 a-1243$ & 0:49:41.98 & $-73: 09: 16.3$ & 15.89 & 15.11 & 3.617 & $1 O$ & $11-02923$ & 05-111479 \\
\hline 00-1754 & $0: 43: 46.43$ & $-73: 07: 02.2$ & 15.73 & 15.04 & 3.619 & $1 O$ & 00-10466 & 03-088641 \\
\hline
\end{tabular}


Table 4. Cepheids (continued).

\begin{tabular}{lllllllll}
\hline Name & RA $(2000)$ & Dec $(2000)$ & $V$ & $I$ & $P[\mathrm{~d}]$ & mode & EROS & OGLE \\
\hline $05-1948$ & $0: 53: 16.69$ & $-73: 21: 14.7$ & $16.36 ?$ & - & 3.915 & $?$ & $05-06269$ & $06-180098$ \\
13-2299 & $0: 53: 27.50$ & $-73: 06: 41.9$ & 16.16 & 15.57 & 4.062 & $F$ & $13-08730$ & $06-199605$ \\
13-1465 & $0: 53: 49.26$ & $-73: 10: 02.3$ & 16.16 & 15.45 & 4.299 & $F$ & $13-05760$ & $06-272411$ \\
05-0885 & $0: 53: 30.84$ & $-73: 17: 38.7$ & 16.11 & 15.33 & 5.592 & $F$ & $05-10015$ & $06-185344$ \\
05-2187 & $0: 52: 53.82$ & $-73: 18: 51.5$ & 16.11 & 15.16 & 7.679 & $F$ & $05-08262$ & $06-099943$ \\
11a-1178 & $0: 49: 55.17$ & $-73: 09: 15.5$ & 15.28 & 14.41 & 9.193 & $F$ & $11-03147$ & $05-111455$ \\
09-0252 & $0: 46: 38.91$ & $-72: 59: 18.2$ & 15.14 & 14.41 & 10.442 & $F$ & $09-09108$ & $04-081939$ \\
11b-0094 & $0: 50: 55.67$ & $-73: 12: 12.5$ & 14.61 & - & 15.631 & $F$ & $11-01216$ & $05-271051$ \\
11a-0564 & $0: 49: 44.54$ & $-73: 08: 22.8$ & 14.84 & 13.92 & 15.830 & $F$ & $11-03847$ & $05-117410$ \\
$09-0525$ & $0: 47: 10.64$ & $-72: 57: 37.9$ & 14.23 & - & 23.990 & $?$ & & $04-130978$ \\
\hline
\end{tabular}

Table 5. EROS $2 O$ mode candidates.

\begin{tabular}{lllllllll}
\hline Name & RA $(2000)$ & Dec $(2000)$ & $V$ & $I$ & $P[\mathrm{~d}]$ & mode & EROS & OGLE \\
\hline 15a-2588 & $0: 57: 27.08$ & $-73: 14: 04.8$ & 17.91 & 17.47 & 0.614 & 1 & $15-05054$ & $07-217912$ \\
09-2951 & $0: 46: 46.98$ & $-72: 58: 37.9$ & 17.93 & 17.38 & 0.625 & 1 (DMC) & $09-09899$ & $04-82181$ \\
15b-2278 & $0: 58: 06.51$ & $-73: 18: 24.1$ & 18.14 & 17.53 & 0.634 & 1 & $15-01505$ & \\
05-0432 & $0: 53: 10.59$ & $-73: 19: 21.2$ & 17.89 & 17.38 & 0.657 & 1 (DMC) & $05-08048$ & $06-185268$ \\
$15 \mathrm{~b}-1275$ & $0: 57: 39.91$ & $-73: 16: 37.3$ & 17.64 & 17.21 & 0.706 & 1 & $15-02844$ & \\
$13-0230$ & $0: 53: 39.50$ & $-73: 09: 20.9$ & 17.74 & 17.25 & 0.719 & 1 (DMC) & $13-06286$ & $06-195011$ \\
$11 \mathrm{a}-0043$ & $0: 49: 14.85$ & $-73: 11: 01.3$ & 17.35 & 16.92 & 0.756 & 1 & $11-00673$ & $05-111620$ \\
$11 \mathrm{~b}-1787$ & $0: 50: 57.98$ & $-73: 10: 21.0$ & 17.34 & 16.81 & 0.913 & 1 & $11-3122$ & $05-271293$ \\
$09-0343$ & $0: 47: 10.70$ & $-72: 58: 44.6$ & 17.63 & 17.10 & 1.003 & $1(\mathrm{DMC})$ & $09-10210$ & $04-131229$ \\
$00-2525$ & $0: 43: 39.43$ & $-73: 06: 22.0$ & 17.22 & 16.67 & 1.109 & 1 & $00-10966$ & $03-088717$ \\
\hline
\end{tabular}
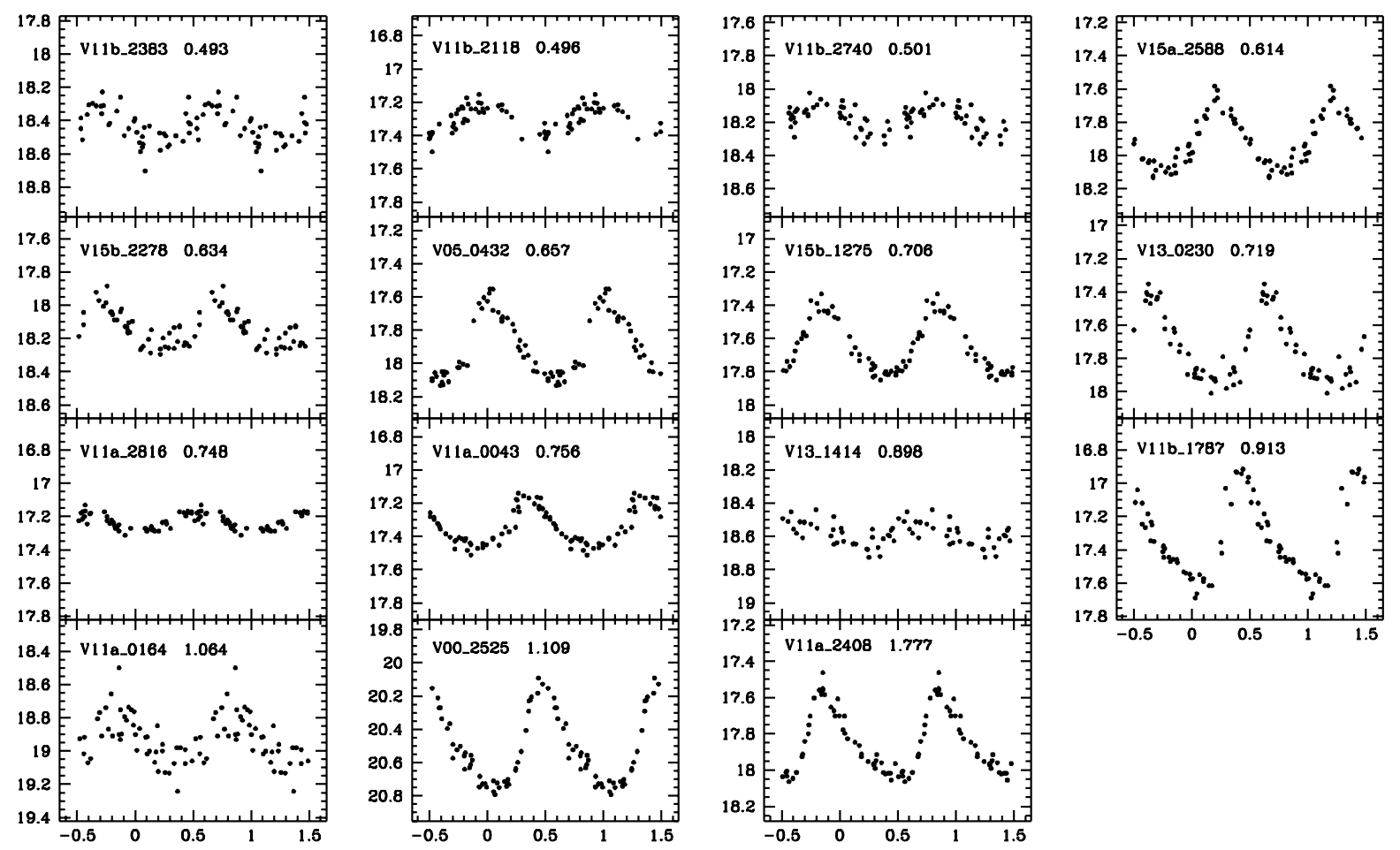

Fig. 2. $V$ light curves of the $2 O$ candidates in our sample, and of the Cepheids not detected by EROS and OGLE (see text); the name and $P$ of each star are reported.

discovered. We have marked with an asterisk the objects that, according to EROS data, were suspected $2 O$ pulsators, while the question mark indicates uncertain pulsation mode according to the Fourier analysis of our data. The equatorial coordinates were derived by con- sidering the stars of our fields which were listed in the USNO Catalog (Monet et al. 1998). The agreement between the derived coordinates and those of the Catalog is better than $1^{\prime \prime}$. In this way we were able to verify that 66 and 52 objects were in common with the EROS 


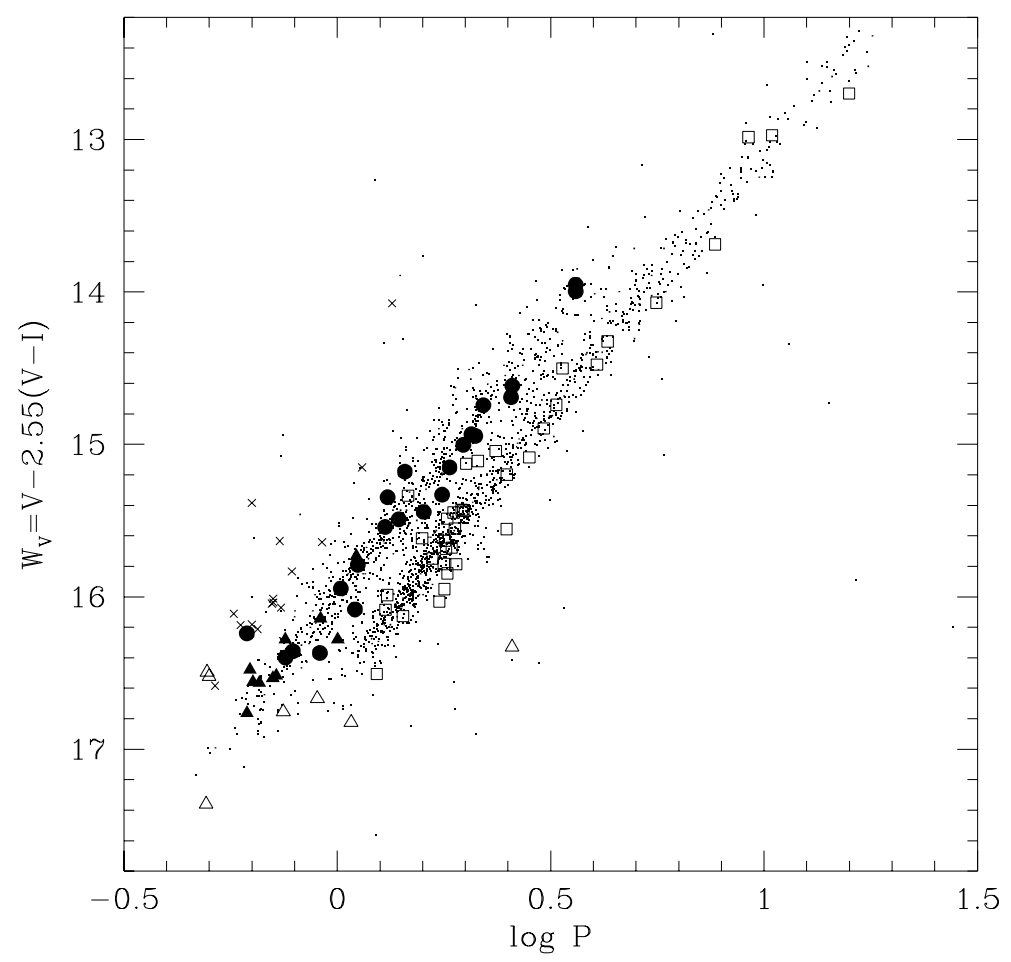

Fig. 3. $P L$ diagram of the Cepheids in our sample, classified according to the light curve shape (Fourier parameters), and compared with OGLE stars. Open squares: F Cepheids; filled circles: $1 O$ Cepheids; filled triangles: EROS $2 O$ candidates; open triangles: stars with uncertain mode; dots: OGLE $F$ and $1 O$ Cepheids; crosses: OGLE $2 O$ candidates.

and OGLE projects, respectively. The periods derived by us are generally in agreement with those derived by the EROS and OGLE projects; the differences are generally less than $10^{-4} \mathrm{~d}^{-1}$, which is the expected accuracy from our data baseline. Only four Cepheids show larger period discrepancies: V05-1050 ( $\left.P_{\mathrm{OGLE}}=1.967 \mathrm{~d}\right)$, V05$1948\left(P_{\text {OGLE }}=3.941 \mathrm{~d}\right.$; the star is very close to the border of our field, and only 16 measurementes are available), V13-2299 ( $\left.P_{\text {OGLE }}=4.062 \mathrm{~d}\right), \mathrm{V} 13-1465\left(P_{\mathrm{OGLE}}=\right.$ $4.299 \mathrm{~d}$ ); we adopted the OGLE value, owing to the better data baseline. The $V$-light curves of the suspected $2 O$ and the newly discovered Cepheids (i.e. not observed by EROS and OGLE projects) are plotted in Fig. 2. The average $V$ and $I$ magnitudes were derived by fitting the data with truncated Fourier series containing the appropriate number of harmonics for each star. The $I$ colours of V11b-0094 and V09-0525 are missing because their images are saturated in our frames. The adopted formula for the Fourier decomposition was

$V=V_{0}+\sum A_{i} \cos \left[2 \pi i f\left(t-T_{0}\right)+\phi_{i}\right]$,

and the derived Fourier parameters are the phase differences $\phi_{i 1}=\phi_{i}-i \phi_{1}$ and amplitude ratios $R_{i 1}=A_{i} / A_{1}$. The Fourier parameters were used to determine the pulsation mode of the stars, and it is reported in Tables 3 and 4. It is generally coincident with that obtained by OGLE; the exception is discussed in the following along with another few discrepant cases. The Cepheids are plotted in the $P L$ diagram shown in Fig. 3, along with OGLE stars; with few exceptions, $F$ and $1 O$ Cepheids discriminated on the basis of the Fourier parameters are well separated in the $P L$ diagram.

V11b-2129 appears to be a very red star, while according to OGLE it should be a normal Cepheid. After an inspection of our images, it was possible to conclude that our I measurements are affected by the presence of a faint star, which appears to be resolved in the $V$ frames; in the subsequent discussion this object was not more considered.

V15a-1156 has $F$ mode Fourier parameters, but in the $P L$ diagram it is located among the $1 O$ mode pulsators. Differently from $F$ mode pulsators with similar $P$, it has a significantly low amplitude, $\Delta V<0.4$, therefore we think it has a relatively bright companion.

V11a-2411 was classified as $1 O$ by OGLE. The Fourier parameters of the $V$-light curve are intermediate between those of $F$ and $1 O$ mode; given the position in the $P L$ diagram we consider this star a $F$ mode pulsator.

V11a-0262 was classified as FA by OGLE, i.e. a fainter star than normal Cepheids; it has a relatively blue color, $V-I=0.32$, which explains the larger $W_{V}$ value and the position in the $P L$ diagram. This and other similar stars will be discussed in another paper.

We detected moreover a new double-mode Cepheid (DMC), V11b-2003, which has $P$ ratio of 0.760 , therefore the two modes should be the $1 O$ and the $F$ one; also the EROS data suggest that it is a DMC. On the other hand, our data are not sufficient to get evidence of the second mode of V05-0076, which is a known DMC. Other DMCs are discussed in the next section. 

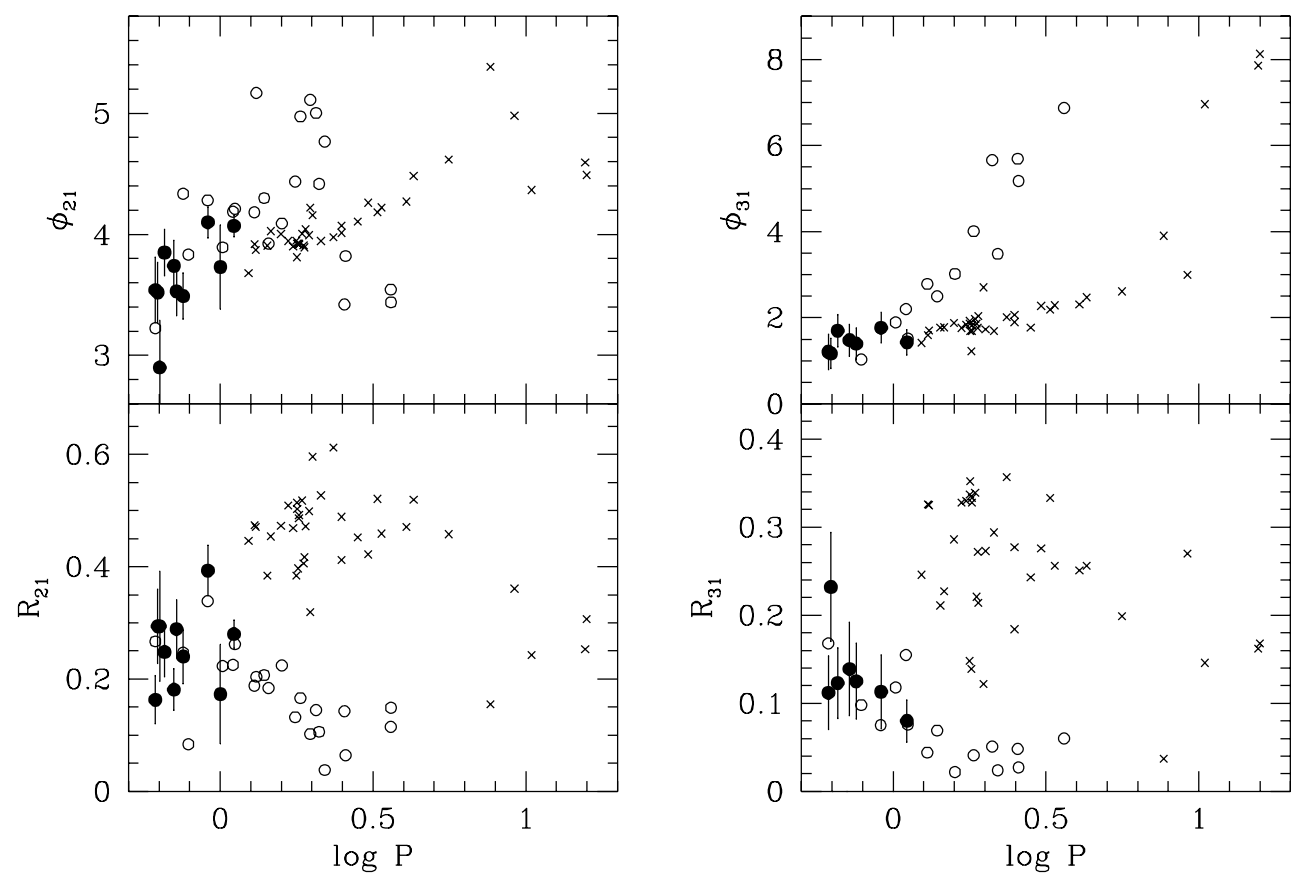

Fig. 4. $V$ band Fourier parameters of EROS $2 O$ candidates (filled circles) compared with other SMC F (crosses) and $1 O$ (circles) mode Cepheids in our sample.

Table 6. Fourier parameters of EROS $2 O$ candidates.

\begin{tabular}{llllllll}
\hline Star & $P$ & s.d. & $A_{1}$ & $R_{21}$ & $\phi_{21}$ & $R_{31}$ & $\phi_{31}$ \\
\hline 15a-2588 & .614 & .037 & .209 & .163 & 3.54 & .112 & 1.21 \\
& & & .009 & .043 & 0.27 & .042 & 0.41 \\
09-2951 & .625 & .065 & .205 & .294 & 3.52 & .232 & 1.17 \\
& & & .061 & .066 & 0.25 & .062 & 0.35 \\
15b-2278 & .634 & .051 & .122 & .294 & 2.90 & - & - \\
& & & .012 & .098 & 0.39 & - & - \\
05-0432 & .657 & .039 & .234 & .248 & 3.85 & .123 & 1.70 \\
& & & .009 & .044 & 0.19 & .041 & 0.38 \\
15b-1275 & .706 & .032 & .207 & .181 & 3.74 & - & - \\
& & & .007 & .037 & 0.21 & - & - \\
13-0230 & .719 & .049 & .247 & .289 & 3.53 & .139 & 1.48 \\
& & & .012 & .052 & 0.20 & .053 & 0.37 \\
11a-0043 & .756 & .027 & .141 & .240 & 3.49 & .125 & 1.40 \\
& & & .006 & .048 & 0.19 & .043 & 0.37 \\
11b-1787 & .913 & .048 & .303 & .393 & 4.10 & .113 & 1.77 \\
& & & .012 & .045 & 0.13 & .043 & 0.36 \\
09-0343 & \multirow{2}{*}{1.003} & .036 & .167 & .173 & 3.73 & - & - \\
& & & .019 & .089 & 0.35 & - & - \\
00-2525 & \multirow{2}{*}{1.109} & .029 & .293 & .280 & 4.07 & .080 & 1.43 \\
& & & .007 & .025 & 0.09 & .024 & 0.30 \\
\hline
\end{tabular}

\section{EROS $2 O$ mode candidates}

The EROS $2 O$ candidates are listed in Tables 5 and 6 along with their relevant parameters. $2 O$ mode Cepheids could be discriminated by means of $P, L$, Fourier parameters (and amplitudes). The Fourier parameters of the $V$ light curves of the candidates are plotted in Fig. 4, along with those of the other Cepheids observed by us. Apart possibly from 15b-2278, which has a small but uncertain $\phi_{21}$ value, none of the ten candidates appear to have very different parameters from those of $1 O$ Cepheids. Moreover, as regards $P$ and $L$, all the candidates lay on the sequence of first overtone pulsators (Fig. 3).

A careful search for multimode pulsation was then performed. The stars 09-2951 and 09-0343 turned out to be $2 O / 1 O$ DMCs with the $1 O$ as main pulsation mode; this is in agreement with OGLE results. On the other hand, we failed to detect the multimode pulsation of 13-0230, which is another known OGLE 2O/1O DMC. After an analysis of both our and OGLE $I$ data sets, one candidate, 05-0432 (previously classified as $1 O$ by OGLE), turned out to be a $2 O / 1 O \mathrm{DMC}$ with $P_{1}=0.656560 \pm .000007 \mathrm{~d}$, $P_{2}=0.52918 \pm .00003 \mathrm{~d}$, and period ratio 0.80599 . The second overtone component has a very small amplitude. The relevant parameters of the new DMC are reported in Table 7, along with those of 09-2951 and 09-0343 derived by us. Since the main component is the $1 O$ mode, while the $2 O$ mode is generally weak, the DMC nature of these four stars has a negligible effect on the parameters plotted in Fig. 4.

Taking into account all the available data, we conclude that the EROS $2 O$ candidates are very probably $1 O$ pulsators or DMCs with the $1 O$ mode as main component. Owing to the position in the $P L$ diagram, two variable stars with very short $P$ discovered by us, $11 \mathrm{~b}-2118$ and 11b-2740, could be considered suspected $2 O$ pulsators, in particular the latter. The low amplitude and data quality however do not allow us to derive reliable Fourier parameters.

\section{OGLE $2 O$ mode candidates}

OGLE database is a very rich mine and we tried to dig out some results concerning a detailed description 
Table 7. Relevant data for the DMCs found among the EROS $2 O$ candidates.

\begin{tabular}{lcrlllllllll}
\hline Star & band & $P_{1}$ & $A_{1}$ & $R_{21}$ & $\phi_{21}$ & $P_{2}$ & $A_{1}$ & $R_{21}$ & $\phi_{21}$ & $P_{2} / P_{1}$ & remarks \\
\hline $09-2951$ & $I$ & .6248 & .129 & .234 & 3.68 & .5040 & .039 & .114 & 4.65 & .8067 & \\
& & & .003 & .021 & 0.10 & & .003 & .070 & 0.60 & & \\
$05-0432$ & $I$ & .6566 & .145 & .271 & 3.82 & .5292 & .023 & - & - & .8060 & New DMC \\
& & & .003 & .019 & 0.08 & & .003 & - & - & & \\
$09-0343$ & $I$ & \multirow{2}{*}{1.0029} & .103 & .210 & 4.18 & .8040 & .027 & .223 & 5.28 & .8017 & \\
& & & .002 & .018 & 0.09 & & .002 & .065 & 0.31 & & \\
\hline
\end{tabular}

Table 8. Fourier decomposition coefficients of some OGLE pure $2 O$ Cepheid candidates.

\begin{tabular}{llllllll}
\hline OGLE n. & $P$ & s.d. & $A_{1}$ & $R_{21}$ & $\phi_{21}$ & $R_{31}$ & $\phi_{31}$ \\
\hline 1146599 & .702 & .017 & .040 & $(.002)$ & - & .122 & 1.20 \\
& & & .002 & - & - & .049 & 0.43 \\
5235321 & .737 & .016 & .033 & .124 & 1.97 & .106 & 6.27 \\
& & & .002 & .052 & 0.44 & .052 & 0.51 \\
693 & .921 & .014 & .033 & $(.035)$ & - & .082 & 3.44 \\
& & & .001 & - & - & .044 & 0.57 \\
617477 & .572 & .021 & .032 & .126 & 2.21 & .125 & 2.15 \\
& & & .002 & .067 & 0.56 & .067 & 0.59 \\
\hline
\end{tabular}

of $2 O$ Cepheid light curves, taking also into account the higher order terms of Fourier decomposition. In the present paper we consider some of the properties of the light curve fit up to the third order in relation to the problem of $2 O$ pulsations. Firstly we performed an analysis of $I$ light curves of 15 pure $2 O$ Cepheid candidates (Udalski et al. 1999a). In agreement with these authors, for 6 stars the Fourier parameters were too uncertain, that is the $R_{21}$ value is smaller than or comparable with the respective error, and for 5 stars only a second-order fit was obtained. For 2 stars, namely SMC SC5 235321 and SMC SC6 17477, it was possible to get a reasonable third order fit, while for another 2 stars, SMC SC6 93 and SMC SC11 46599, a significant third-order term was derived in spite of a negligible second-order term. The results for the last four stars are reported in Table 8 .

It is instructive to give a look at the shape of the light curves of a sample of the candidates (Fig. 5). Even if the amplitude is very small (between 0.06 and $0.1 \mathrm{mag}$ ), the large number of observed points per star allows us to derive with confidence some conclusions about the light curve shapes. Several stars have a light curve which is not very sinusoidal, and bumps and asymmetries can be noted. In particular, while SMC SC4 97000 has a featureless light curve, SMC SC11 46599 has a bump on the descending branch, and SMC SC6 93 and SMC SC5 235321 have a bump on the ascending branch. These features explain the large range of phase difference values, which are typical of the known resonance effects in $F$ and $1 O$ mode Cepheids with $P$ near $10 \mathrm{~d}$ and $3 \mathrm{~d}$, respectively.

Unfortunately, the small number of stars and the uncertainty in the Fourier parameters do not allow us to suggest observationally a reliable resonance center or period for $2 O$ Cepheids. We have also considered 2O/1O double mode Cepheids in SMC (Udalski et al. 1999b) and LMC

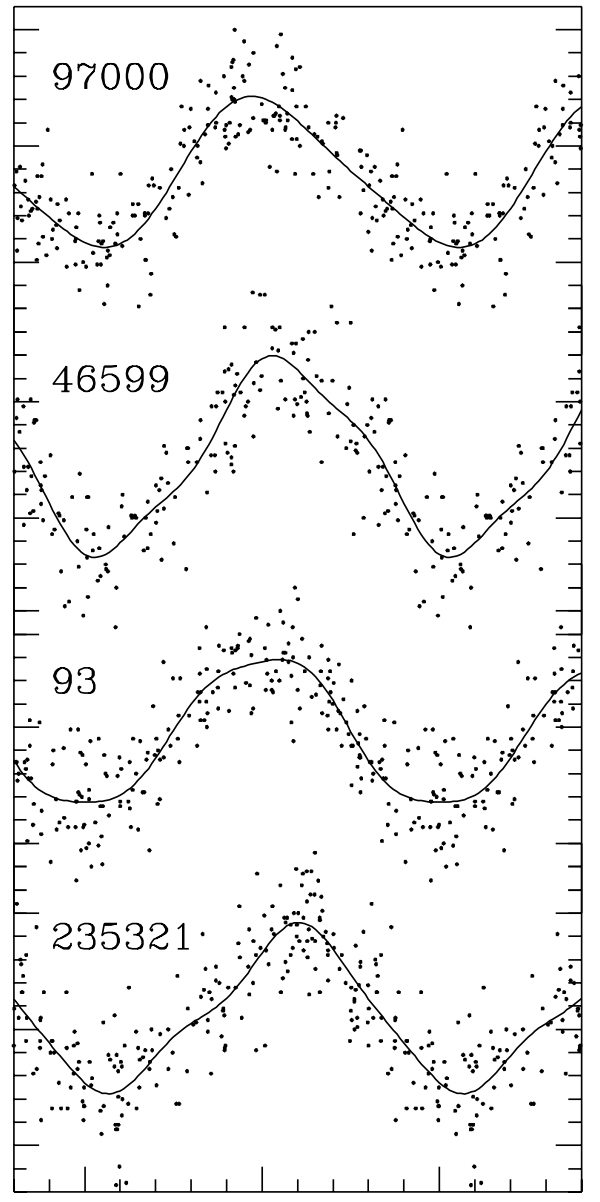

Fig. 5. Sample of $I$ light curves of OGLE pure $2 O$ Cepheid candidates, phased with the respective $P$; the small ticksize of ordinatae is $0.01 \mathrm{mag}$.

(Soszynski et al. 2000) and the respective periods reported in the database. The $I$ light curves were analysed taking into account initially all the cross coupling terms up to the third order. A procedure for discarding progressively the less significant components was introduced and the final frequency set of each star contained only terms with amplitude larger than $2 \sigma$, where $\sigma$ is the error in the amplitude. The phase differences and amplitude ratios were computed for the $1 O$ and $2 O$ components, respectively, and they are plotted in Fig. 6. For comparison purposes, we analyzed also $F$ and $1 O$ mode light curves of LMC (Udalski et al. 1999c) and SMC (Udalski et al. 1999d) Cepheids, and the results are plotted in the same figure. For clarity reasons, the error bars are plotted only in one of 
Table 9. Results of simulations of blending effect on Fourier parameters.

\begin{tabular}{ccccccccccc}
\hline$L_{\text {comp }} / L_{\text {ceph }}$ & $R_{21}$ & $R_{31}$ & $R_{41}$ & $R_{51}$ & $\phi_{21}$ & $\phi_{31}$ & $\phi_{41}$ & $\phi_{51}$ & $\Delta V$ & $<V_{\text {ceph }}>-<V>$ \\
\hline 0.0 & .450 & .250 & .115 & .042 & 4.058 & 2.020 & 5.887 & 3.250 & 1.00 & 0 \\
0.1 & .454 & .253 & .119 & .045 & 4.038 & 1.977 & 5.828 & 3.186 & .91 & 0.11 \\
0.2 & .458 & .256 & .122 & .047 & 4.020 & 1.943 & 5.780 & 3.134 & .84 & 0.21 \\
0.5 & .467 & .263 & .130 & .053 & 3.985 & 1.867 & 5.678 & 3.021 & .68 & 0.47 \\
1.0 & .476 & .270 & .137 & .059 & 3.951 & 1.792 & 5.577 & 2.910 & .51 & 0.78 \\
2.0 & .486 & .278 & .146 & .066 & 3.918 & 1.718 & 5.479 & 2.800 & .35 & 1.23 \\
\hline
\end{tabular}
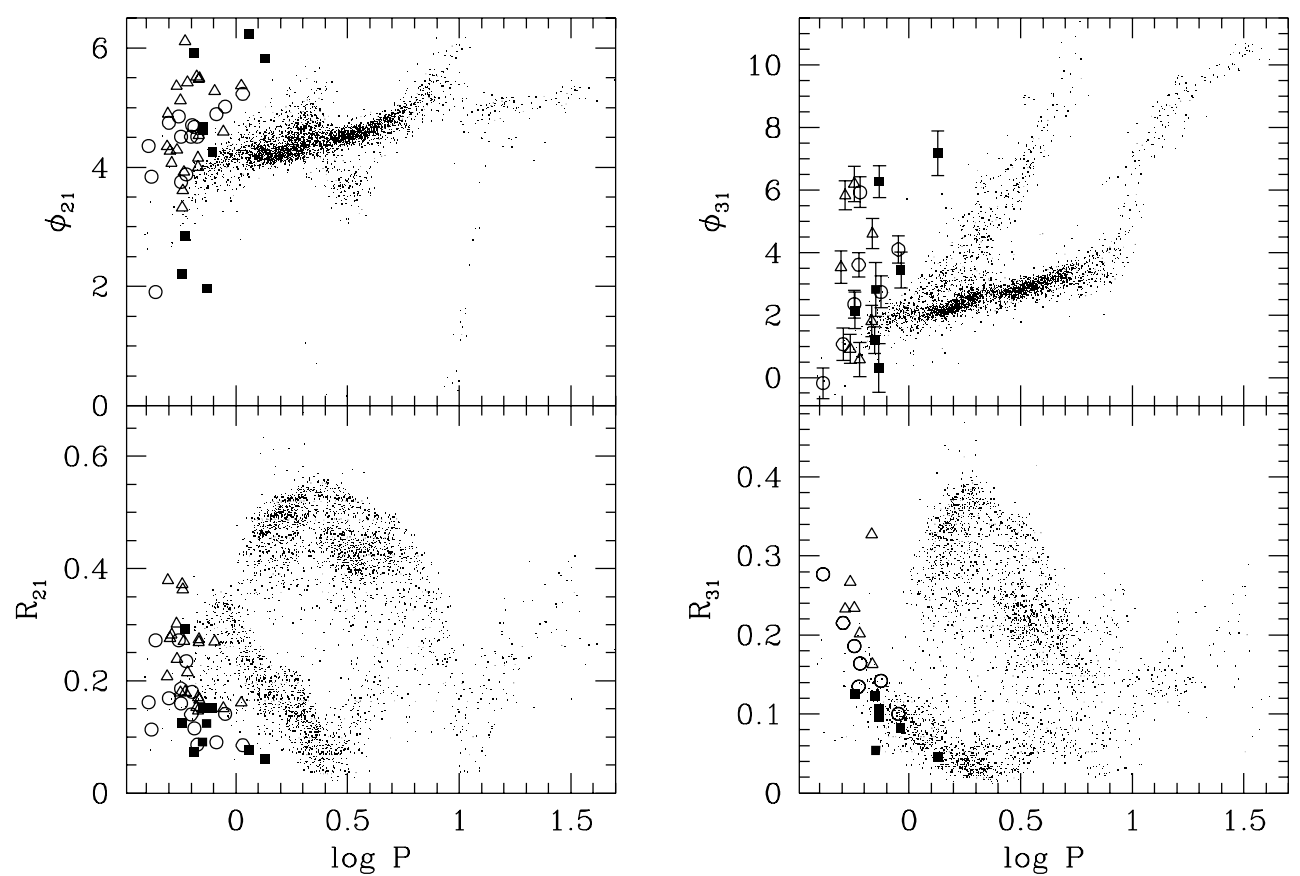

Fig. 6. $I$ band Fourier parameters of OGLE pure $2 O$ SMC Cepheids (filled squares) and $2 O$ component of double mode SMC (triangles) and LMC (circles) Cepheids, compared with $F$ and $1 O$ SMC and LMC Cepheids (dots).

the panels. The OGLE pure $2 O$ candidates plotted in the diagrams are only those with reliable Fourier parameters.

\section{Discussion and conclusion}

The referee of this paper pointed out the need to discuss the possible effects of blending on the light curves. This could be the case since the very low $W_{V}$ value (Fig. 3) of some OGLE $2 O$ candidates would indicate a blue companion. It is well known that a Cepheid in a binary system, with a companion of similar luminosity, has an observed light curve amplitude which is lower than its intrinsic value; the same occurs in case of an apparent close companion (see e.g. Sect. 4 for the star V15a-1156). To the best of our knowledge an adequate discussion of the effects on the Fourier parameters is lacking, even if it is known that those on the lower order parameters are negligible. We have considered a typical light curve of a Cepheid pulsating in the $F$ mode; the results for a $1 O$ Cepheid light curve are similar. We simulated its changes for various ratios of the luminosity of the companion $\left(L_{\text {comp }}\right)$ to the luminosity of the Cepheid $\left(L_{\mathrm{ceph}}\right)$, and the results of the Fourier decomposition are reported in Table 9, which contains the $L_{\text {comp }} / L_{\text {ceph }}$ ratio, amplitude ratios, phase differences, the light curve amplitude and the difference between the magnitude of the Cepheid and that of the system. For increasing $L_{\text {comp }}$ the amplitude ratios tend to increase and the phase differences to decrease, and the effect is larger for higher order parameters. These changes are related to the nonlinearity of the relation between intensity and magnitude. However, taking into account the scatter of the distribution of Fourier parameters vs. $P$ of the observed Cepheids, we can confirm that the blending effect on the lower order parameters is negligible. We have performed several simulations by changing the number of the points and assuming various errors on the measurements. The results essentially do not change, within the expected formal errors (as long as the light curve quality is acceptable), and increasing $L_{\text {comp }}$ implies decreasing observed pulsation amplitude and decreasing order of reliable fit (i.e. number of significant parameters). A possible color effect (related to the bandwith of the filter) must be considered negligible too. The conclusion of these tests is that the lower order Fourier parameters can confidently be 
used as a pulsation mode discriminator even in the case of blending; this is clearly a powerful property when dealing with Cepheids with short $P$ in external galaxies.

Looking at Fig. 6, we can say that pure $2 O$ Cepheids and the $2 O$ component of DMCs behave in a similar way to $F$ and $1 O$ Cepheids. In the case of the amplitude ratios, the trends are roughly similar to those of $1 O$ and $F$ mode stars, but they are displaced towards shorter $P$. The $\phi_{21}$ values are intrinsically scattered (i.e. the errors are relatively small), and it is not possible to see smooth changes in their trend as in $1 O$ and $F$ mode cases. In the $\phi_{31}-\log P$ panel, $1 O$ and $F$ mode Cepheids form nicely two parallel sequences near $\log P \sim 0.5$ and $\sim 1$, respectively. The two sequences are related of course to the same resonance phenomena between pulsation modes which are responsible of the trends in $\phi_{21}$ and $R_{21}$. It is not possible to conclude confidently the same for $2 O$ mode stars at $\log P \sim-0.2$, even if the (intrinsic) scatter appears to be significant.

On the whole we think that the trends and scatter of $2 O$ mode parameters in Fig. 6 in comparison with $1 O$ and $F$ ones are indicating that also in $2 O$ Cepheids a resonance is probably operating, with a resonance center localized between $\log P \sim-0.2$ and $\sim 0$, which would confirm the predictions of the models. The scatter seen in the phase difference values is intrinsic and is not simply related to the errors in the estimates, nor could it be explained by the presence of some pulsating stars among pure $2 O$ mode Cepheids whose nature has not been identified correctly. Unlike $1 O$ and $F$ mode Cepheids, however, the $2 O$ mode ones probably have a limited mass (and hence $P$ ) range, and a larger sensitivity of the pulsational characteristics, e.g. light curve shapes, to small differences in the physical parameters.

In conclusion, even if we have not found further reliable $2 O$ candidates other than the OGLE ones, we can say that the presently-known candidates tend to support the predicted resonance effect. On the other hand, more accurate data are needed for a definitive confirmation.

Acknowledgements. Thanks are due to the referee J. P. Beaulieu for the useful comments and suggestions.

\section{References}

Alcock, C., Allsman, R. A., Axelrod, T. S., et al. 1995, AJ, 109, 1653

Alcock, C., Allsman, R. A., Alves, D., et al. 1999, ApJ, 511, 185

Antonello, E. 1993, A\&A, 279, 130

Antonello, E., \& Kanbur, S. M. 1997, MNRAS, 286, L33

Antonello, E., Poretti, E., \& Reduzzi, L. 1990, A\&A, 236, 138

Beaulieu, J. P., Grison, P., \& Tobin, W. 1955, A\&A, 303, 137

Beaulieu, J. P. 1997, private communication

Beaulieu, J. P., Krockenberger, M., Sasselov, D. D., et al. 1997, A\&A, 321, L5

Bono, G., Caputo, F., \& Marconi, M. 2001, MNRAS, 325, 1353

Buchler, J. R. 1996, in Variable Stars and the Astrophysical Returns of the Microlensing Surveys, ed. R. Ferlet, J. P. Maillard, \& B. Raban (Éditions Frontières, Paris), 181

Davis, L. E. 1994, A Reference Guide to the IRAF/DAOPHOT Package (IRAF Programming Group, NOAO, Tucson)

Feuchtinger, M., Buchler, J. R., \& Kollath, Z. 2000, ApJ, 540, 1056

Kaluzny, J., Stanek, K. Z., Krockenberger, M., et al. 1998, AJ, 115,1016

Landolt, A. U. 1992, AJ, 104, 340

Mantegazza, L. 1983, A\&A, 118, 321

Mantegazza, L., \& Antonello, E. 1998, A\&AS, 132, 39

Monet, D. G., et al. 1998, USNO-A2.0 (Washington: US Naval Obs.)

Simon, N. R., \& Lee, A. S. 1981, ApJ, 248, 291

Soszynski, I., Udalski, A., Szymanski, M., et al. 2000, Acta Astron., 50, 451

Stetson, P. B. 1987, PASP, 99, 191

Stobie, R. S. 1969, MNRAS, 144, 461

Tody, D. 1993, in Astronomical Data Analysis Software and Systems II, ed. R. J. Hanisch, R. J. V. Brissenden, \& J. Barnes, PASPC, 52, 173

Udalski, A., Soszynski, I., Szymanski, M., et al. 1999a, Acta Astron., 49, 1

Udalski, A., Soszynski, I., Szymanski, M., et al. 1999b, Acta Astron., 49, 45

Udalski, A., Soszynski, I., Szymanski, M., et al. 1999c, Acta Astron., 49, 223

Udalski, A., Soszynski, I., Szymanski, M., et al. 1999d, Acta Astron., 49, 437 